\title{
Neural competition through visual similarity in face selection.
}

Citation for published version (APA):

Gentile, F., \& Jansma-Schmitt, B. M. (2010). Neural competition through visual similarity in face selection. Brain Research, 1351, 172-184. https://doi.org/10.1016/j.brainres.2010.06.050

Document status and date:

Published: 10/09/2010

DOI:

10.1016/j.brainres.2010.06.050

Document Version:

Publisher's PDF, also known as Version of record

Document license:

Taverne

Please check the document version of this publication:

- A submitted manuscript is the version of the article upon submission and before peer-review. There can be important differences between the submitted version and the official published version of record.

People interested in the research are advised to contact the author for the final version of the publication, or visit the DOI to the publisher's website.

- The final author version and the galley proof are versions of the publication after peer review.

- The final published version features the final layout of the paper including the volume, issue and page numbers.

Link to publication

\footnotetext{
General rights rights.

- You may freely distribute the URL identifying the publication in the public portal. please follow below link for the End User Agreement:

www.umlib.nl/taverne-license

Take down policy

If you believe that this document breaches copyright please contact us at:

repository@maastrichtuniversity.nl

providing details and we will investigate your claim.
}

Copyright and moral rights for the publications made accessible in the public portal are retained by the authors and/or other copyright owners and it is a condition of accessing publications that users recognise and abide by the legal requirements associated with these

- Users may download and print one copy of any publication from the public portal for the purpose of private study or research.

- You may not further distribute the material or use it for any profit-making activity or commercial gain

If the publication is distributed under the terms of Article $25 \mathrm{fa}$ of the Dutch Copyright Act, indicated by the "Taverne" license above, 


\title{
Research Report
}

\section{Neural competition through visual similarity in face selection}

\author{
F. Gentile*, B.M. Jansma \\ Department of Cognitive Neuroscience, Faculty of Psychology and Neuroscience, Maastricht University, The Netherlands, \\ Maastricht Brain Imaging Center (M-BIC), Maastricht University, The Netherlands
}

\section{A R T I C L E I N F O}

Article history:

Accepted 21 June 2010

Available online 1 July 2010

Keywords:

Face perception

Neural competition

Attention

Visual similarity

Face selective areas

fMRI

\begin{abstract}
A B S T R A C T
Ignoring and selecting relevant faces has a strong impact in everyday life. We often perform tasks where faces may be considered irrelevant (e.g. having a drink in a crowded bar) or tasks where we need to filter out all but one face (e.g. searching for a friend in a crowd). The present study was designed to test Biased Competition (BC) as a suitable model for selection in the context of face processing, using functional magnetic resonance imaging (fMRI). Pairs of similar or dissimilar faces were presented simultaneously, and subjects had to attend to one face (target face) or ignore both faces. According to the BC model, faces simultaneously presented compete for representation. Spatial attention biases these competitive interactions towards neural processing of the target face only. We compared fMRI signal changes related to the processing of dissimilar or similar faces in the attend-to-face and ignore-faces task. In the ignore condition we expected that similar faces would compete more than dissimilar faces as similar features (faces) are supposed to be encoded by the same population of neurons resulting in a lower fMRI signal change in face selective areas. The BC model also predicts an enhancement of the fMRI signal change for attend-to-face vs. ignore-faces condition, regardless of the degree of the similarity between the two faces. Both hypotheses were confirmed by the data, indicating $\mathrm{BC}$ as a possible selection mechanism within the fusiform face area (FFA) and occipital face area (OFA) for face stimuli.
\end{abstract}

() 2010 Elsevier B.V. All rights reserved.

\section{Introduction}

The visual system receives many stimuli at the same time. Attentional mechanisms are needed to guide visual processing for relevance (Broadbent, 1958; Desimone and Duncan, 1995; Treisman, 1969). It is widely assumed that attention improves processing of behaviorally relevant objects (Carrasco et al., 2004; Hawkins et al., 1990; Luck et al., 1994). According to the influential spotlight metaphor, attention enhances the neural response for stimuli presented at the relevant location (Brefczynski and DeYoe, 1999; Hillyard and Munte, 1984; Luck et al., 1994; Posner et al., 1980; Reynolds et al., 2000).
Next to its relevance in optimizing visual processing (facilitation), spatial attention also plays a crucial role in segregating one item from a cluster of irrelevant objects (selection). Monkey cell recording studies investigated the effect of selective attention in the ventral and dorsal visual stream. In these experiments, a pair of items was presented at the same time within the receptive field of the target neurons. When stimuli were ignored, the neural response to both items was a weighted average of the response elicited by each of the stimuli alone. However, when attention was shifted to one of the two objects, the response to the pair was driven preferentially by the attended stimulus (Chelazzi et al., 1998,

\footnotetext{
* Corresponding author. Department of Cognitive Neuroscience, Faculty of Psychology and Neuroscience, Maastricht University, P.O. Box 616, 6200 MD Maastricht, The Netherlands. Fax: +31 433884125.

E-mail address: f.gentile@maastrichtuniversity.nl (F. Gentile).
} 
2001; Desimone and Duncan, 1995; Luck et al., 1997; Miller et al., 1993; Moran and Desimone, 1985; Reynolds et al., 1999; Treue and Martinez Trujillo, 1999; Treue and Maunsell, 1996). These findings gave rise to models for attentional selection based on competitive interactions between neural representations of stimuli. One of the most influential competitive models is Biased Competition (BC). The BC model states that multiple stimuli that are simultaneously presented to the receptive field of neurons under investigation compete for neural representation (Desimone, 1998; Desimone and Duncan, 1995; Kastner and Ungerleider, 2000, 2001; Pessoa et al., 2003; Reynolds and Desimone, 1999). Local and intracortical connections seem to regulate this mechanism of neural competition, usually observed for ignored stimuli (Reynolds and Desimone, 1999). Attending to a stimulus activates a fronto-parietal network, which biases the competition in favor of the attended item (target), via feedback towards perceptual areas. As a result, neurons that respond to the target stimulus remain active, while neurons that respond to the ignored stimuli are suppressed.

Further evidence in favor of BC in early visual processing has come from human functional magnetic resonance imaging (fMRI) studies using abstract visual objects. BC has been mainly tested by presenting stimuli either sequentially (no competition) or simultaneously (competition) (Beck and Kastner, 2005, 2007; Kastner et al., 1998, 1999, 2001). In these studies, the fMRI signal change was significantly lower for simultaneously presented compared to sequentially presented objects. The authors interpreted this effect as neural suppression, supporting the idea that stimuli compete for representation in the visual cortex. In subsequent experiments, the role of attention within the BC framework was also investigated. In line with the electrophysiological studies in monkeys, human attentional mechanisms also seem to influence neural competition. In particular, fMRI signal change increased either when one stimulus was particularly salient (Beck and Kastner, 2005) or when one of the items was covertly attended to (Bles et al., 2006; Kastner et al., 1999). This enhancement suggested that attention can bias the neural competition and thereby facilitates the selection of the attended item. Note that all these studies used rather abstract stimuli such as Gabor patches, or colorful pattern pictures, and had a focus on early visual processing. In the present study, we tested the BC model in the context of face processing.

Face processing has been largely investigated in humans using electroencephalography (EEG) and fMRI. In particular, event-related potential (ERP) studies showed a significant difference for a negative potential around $170 \mathrm{~ms}$ after stimulus onset (N170) when the presented faces were compared to non-face stimuli. This effect was particularly evident at the lateral occipital electrode sites (Bentin et al., 1996; Rossion et al., 1999). In the last decade, fMRI allowed to localize the source of face processing within a region in the fusiform gyrus, which has become known as the fusiform face area (FFA) (Gauthier et al., 2000; Grill-Spector et al., 2004; Halgren et al., 1999; Haxby et al., 2001; Kanwisher et al., 1997). Furthermore, a combined EEG and magnetoencephalographic study localized the source of the N170 in the fusiform gyrus (Deffke et al., 2007). However, thus far, the neural mechanism underlying selection of one face among several faces within FFA is still unknown.

To our knowledge, only a few studies examined the neural correlates of processing multiple face displays. Jacques and Rossion $(2004,2006)$, recently found that the N170 amplitude was smaller when a center target face was presented in a context of other faces than when it was shown with scrambled faces. This effect, referred to as repetition facilitation (or priming), was related to residual activation of neural representation of the preceding face context in the sequence of trials. Alternatively, the observed effect could be related to sensory suppression, which might result from competitive interactions between the items simultaneously presented.

In the present study, we investigated the mechanism of face selection by means of a bias competition paradigm using fMRI. The high spatial resolution of this technique allowed us to restrict the region of interest to FFA and to examine secondary effects in other regions of the brain. The experimental design consisted of two simultaneously presented faces. They had to be either ignored or attended to. With regard to the ignore-faces task, we assumed that simultaneously presented faces are also processed as such in FFA (Halgren et al., 1999; Reinholz and Pollmann, 2007)-hence competing with each other for representation (Desimone, 1998). The attend-to-face task was introduced as a baseline condition for single-face processing (Recanzone, 2000; Reynolds et al., 1999). We compared the neural activation elicited by both tasks to test the competitive aspects of multiple face encoding. According to the BC model we expected a lower fMRI signal change in FFA for the ignore-faces condition compared to the attend-to-face condition.

Neural competition was further investigated by varying the similarity level of the faces presented (similar, dissimilar). We manipulated similarity in order to draw upon the spatial and functional characteristics of neural populations in FFA. In fact, neural populations in the extrastriate cortex seem to be organized according to the similarity level of the object representations (Op de Beeck et al., 2008; Tanaka, 2003). This hypothesis was recently supported by two fMRI studies (Jiang et al., 2006; Loffler et al., 2005), which reported that in FFA two dissimilar faces are encoded by separate populations of neurons. In contrast, similar faces (Jiang et al., 2006) were encoded by cells that are spatially clustered together. A similar finding was reported for faces sharing the same "identities" (Loffler et al., 2005). At a cell population level, these results suggest that neural competition may vary with the degree of similarity between faces in a pair. Therefore, we expected that neural competition between two face representations would be greater among elements encoded by the same neural population (similar faces) than among elements activating distinct subpopulations of neurons (dissimilar faces). This would result, in turn, in a lower fMRI signal change for similar vs. dissimilar faces within the ignore-faces task.

The bias component of the BC framework was investigated by focusing on the attend-to-face task. According to the BC model, spatial attention towards a specific item in the visual field is supposed to resolve neural competition in favor of the attended item only. In this case we expected no effect of the similarity manipulation. The present experiment was designed in such a way that any sequential priming effect 
(or adaptation in fMRI terms) was kept constant between conditions.

Besides a fine grained investigation of FFA, we were also interested in a more general analysis of the whole brain as other areas may be involved in competition and selection. We expected to find a main effect of attention within clusters in the typical fronto-parietal network, consistently activated in various tasks involving spatially directed attention (Corbetta et al., 1993, 1998; Gitelman et al., 1999; Hopfinger et al., 2000; Nobre et al., 1997; Thomsen et al., 2005).

\section{2. $\quad$ Results}

\subsection{Main experiment}

\subsubsection{Behavioral results}

We computed error rates (ERs) and reaction times (RTs) on the ignore-faces (IGN) and attend-to-face (ATT) task for similar (SIM) and dissimilar (DISS) condition. Mean ERs and RTs were then submitted to a repeated-measures ANOVA with two factors, namely attention and similarity. For RTs, no significant main effects of attention, similarity or interaction were observed. Attention: $(F(1,14)=3.99, p=.066$, mean_reaction time ATT $=629 \mathrm{~ms}, \mathrm{SE}=18$, mean_IGN=605 ms, SE=17). Similarity: $(F(1,14)=2.5, p=.136$, mean_SIM $=619 \mathrm{~ms}, \mathrm{SE}=16$, mean_DISS $=614 \mathrm{~ms}, \mathrm{SE}=16)$. Interaction: $(F(3,14)=3.18$, $p=.096)$. The same holds for error rates. Attention: $(F(1,14)=$ 4.52, $p=.052$, mean_ATT $=27 \%, \mathrm{SE}=4$, mean_IGN $=18 \%, \mathrm{SE}=1)$. Similarity: $(F(1,14)=.01, p=.919$, mean_SIM $=22 \%, \mathrm{SE}=2$, mean_DISS $=23 \%, \mathrm{SE}=3)$. Interaction: $(F(3,14)=.00, p=.957)$.

\subsubsection{Eye-movements}

We used the data recorded in a parallel EEG experiment with an identical procedure (instruction, display, task and timing) to monitor for eye-movements. The electroencephalogram (EEG) was measured in 18 different subjects using tin electrodes (32 channels in total, QuickCap, Neuroscan Inc. Tm). Reference electrodes were positioned at both mastoids (A1, A2). The left mastoid (A1) was used as an online reference for the scalp sites. Horizontal eye movements were recorded with a bipolar montage placed at the left and right canthi (HEOG). Electrode impedance was kept below $5 \mathrm{~K} \Omega$. EEG data were recorded and amplified using Neuroscan SynAmps with a $0.05-30 \mathrm{~Hz}$ bandpass and sampled at 4-ms intervals $(250 \mathrm{~Hz})$. The EEG signal was epoched with an interval of $900 \mathrm{~ms}$, time-locked to the onset of the presentation of the pair of faces. Epochs included a 100-ms prestimulus baseline correction interval and they were 0.3$30 \mathrm{~Hz}$ bandpass filtered (Butterworth, zero phase, $24 \mathrm{~dB} / \mathrm{oct}$ ). Horizontal eye movements were defined as visible step functions in the HEOG signal change.

The amount of horizontal eye movements was on average below $4 \%$ of the total amount of trials for all conditions, and they did not differ significantly for the two tasks (attend-toface, ignore-faces) $(F=3.91, p=.065)$. In practice, one would expect a larger amount of horizontal saccades within the attend-to-face related to overt shifts of attention, this directionality would lead to a one-tailed $p$ of .065/2 $(p=.032)$.
This means that in $4 \%$ of the cases, a difference in eye movement cannot be ruled out. The same analysis performed within the ignore-faces condition did not show any difference in the amount of eye movement between the ignore-similarfaces and ignore-dissimilar-faces condition $(F=.81, p=.379)$, indicating that eye movement most likely do not play a role in explaining the observed similarity effect in this condition.

\subsubsection{Region of interest (ROI) analysis in FFA}

A Random effects (RFX) General Linear Model (GLM) analysis restricted to FFA revealed, bilaterally, a significant interaction of factors attention (ATT vs. IGN) and similarity (SIM vs. DISS) (right FFA: $p=.017$, left FFA: $p=.045$ ), a significant main effect of attention (ATT $>$ IGN) (right FFA: $p=.000$, left FFA: $p=.000$ ) and no main effect of similarity. The interaction, qualitatively visible also in the time course in Fig. 1.a, reflected a larger fMRI signal change when dissimilar faces were presented compared to similar faces in the ignore-faces condition, both in the right and left FFA (IGN_SIM $<$ IGN_DISS) (right FFA: $p=.011$, left FFA: $p=.003)$. No significant differences between SIM and DISS displays within the attend-to-face conditions (ATT_SI$\mathrm{M}=\mathrm{ATT}$ _DISS) were observed in either hemisphere, based on simple effect analysis (Fig. 1.a, Table 1).

\subsubsection{Whole-brain GLM analysis for visual areas}

The whole-brain GLM analysis revealed an interaction of factors attention (ATT vs. IGN) and similarity (SIM vs. DISS) $(p=.007$, corrected) also in the inferior occipital sulcus (corresponding to the lateral occipital sulcus in Talairach coordinates). Such a region has recently been associated with face processing and was identified as the occipital face area (OFA) (Dubois et al., 1999; Gauthier et al., 2000; Halgren et al., 1999; Haxby et al., 1999; Hoffman and Haxby, 2000; Kanwisher et al., 1997; Rossion et al., 2003). A post-hoc analysis restricted to OFA revealed a main effect of attention in the left hemisphere $(p=.005)$. The main effect of attention was significant ( $p=.007$, corrected) also in the inferior part of the right OFA, in the primary visual cortex, the posterior part of the collateral sulcus and within the temporal part of the fusiform gyrus (Fig. 1.b, Table 1).

\subsubsection{Whole-brain GLM: frontal regions}

Within the frontal lobe we distinguished several areas showing a main effect of attention. In particular, the contrast ATT $>$ IGN revealed bilateral activations within the insula $(p=.007$, corrected). Lateralized uniquely on the right hemisphere we found clusters of activations for the same contrast ( $p=.007$, corrected) within the inferior frontal gyrus (IFG), the inferior part of the precentral sulcus and within the medial part of the precentral gyrus. The main effect of similarity or interaction of factors was not significant in any of these or other region within the frontal lobe (Fig. 2, Table 1).

\section{Discussion}

In the present paper we investigated the mechanism of face selection in the context of the BC model of attention, using fMRI. According to $\mathrm{BC}$, neural representations of irrelevant (ignored) 
a)

b)

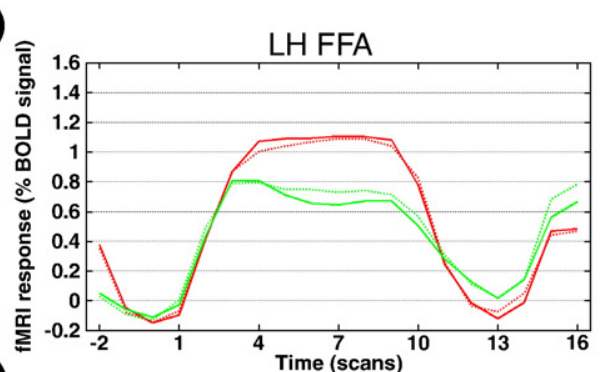

b)

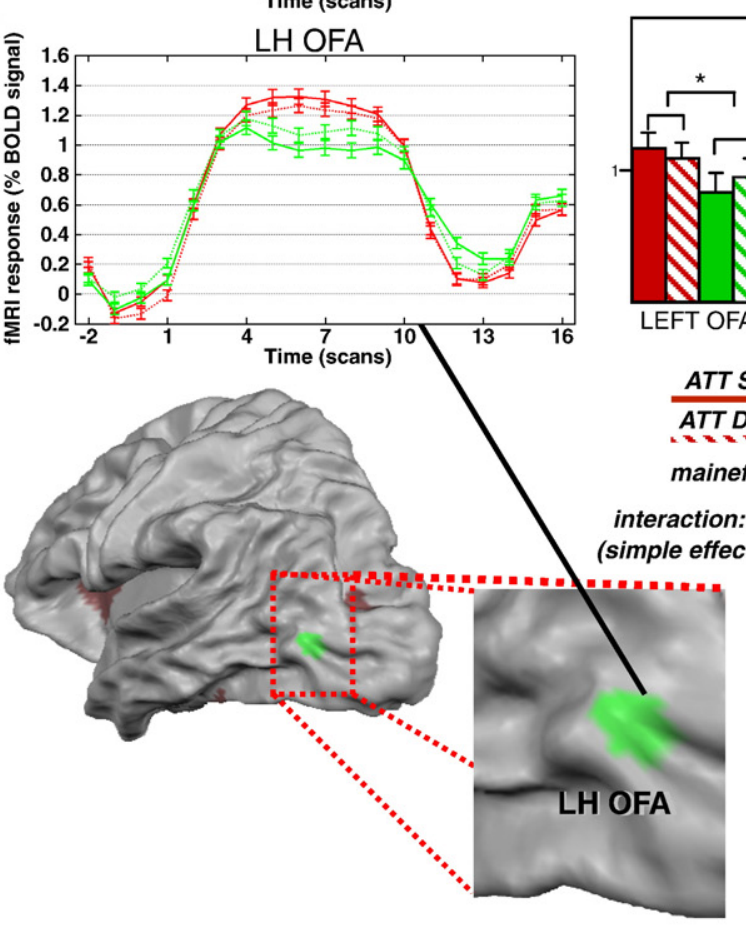

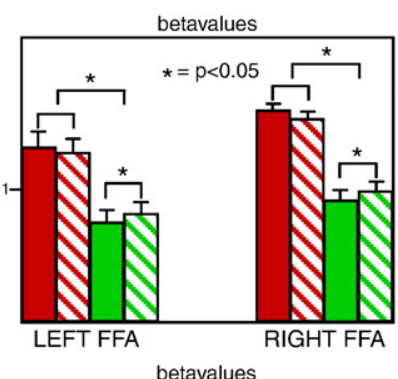

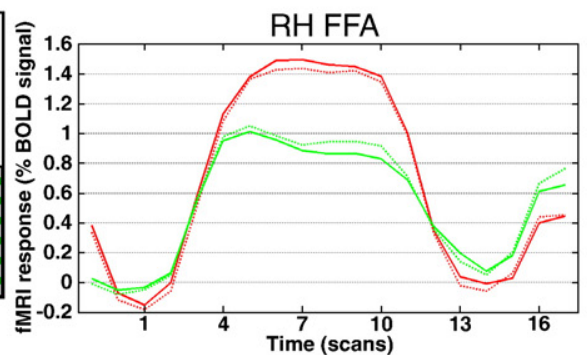

$\star=p<0.05$

RH OFA

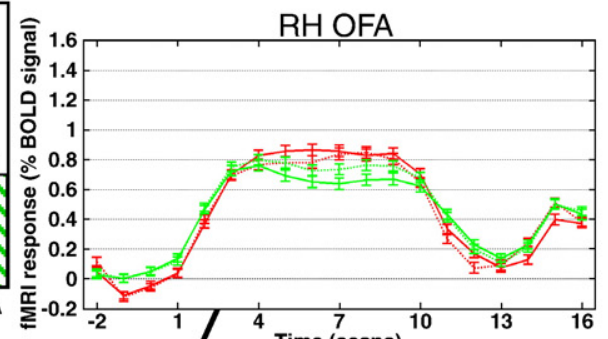

RIGHT OFA

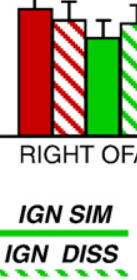

ention

Fig. 1 - Results of the RFX-GLM analysis in (a) FFA (region of interest analysis) and (b) OFA (whole brain analysis), separately for left and right hemisphere. In the central column the beta weights of the GLM analysis in FFA and OFA are displayed. The GLM beta weights related to OFA are referred to the part of OFA showing an interaction effect. The mean time courses of the fMRI signal change per condition and per region of interest are also shown. However, for the mean time course related to FFA error bars are not shown because it results from averaging together mean time courses from region of interest of different size. The error bars in the mean time courses related to OFA represent the standard error (SE). The OFA activation resulting from the whole brain analysis is projected onto the hemispheric surface maps.

Table 1 - Talairach coordinates related to all regions of interest. For FFA the mean Talairach coordinates with the standard deviation are displayed. In fact, the left and right FFA was localized respectively for 14 and 16 subjects, individually.

\begin{tabular}{|c|c|c|c|c|c|c|}
\hline \multirow[t]{2}{*}{ Area } & \multicolumn{3}{|c|}{ Right hemisphere } & \multicolumn{3}{|c|}{ Left hemisphere } \\
\hline & $x$ & $y$ & z & $x$ & $y$ & z \\
\hline FFA & $37 \pm 3$ & $-46 \pm 7$ & $-19 \pm 4$ & $-39 \pm 3$ & $-45 \pm 6$ & $-18 \pm 3$ \\
\hline OFA & 40 & -75 & -3 & -40 & -78 & -3 \\
\hline PrecG & 44 & -12 & 52 & & & \\
\hline PrecS & 38 & -1 & 36 & & & \\
\hline IFG & 45 & 14 & 30 & & & \\
\hline Insula & 32 & 22 & 12 & -30 & 17 & 13 \\
\hline
\end{tabular}

faces engage in mutual suppressive interactions, whereas attention to a particular face biases the competition towards the processing of this face only. Participants were presented with blocks of similar or dissimilar pairs of faces and were asked to attend to one of these faces (attend-to-face condition) or ignore both of them (ignore-faces condition). The direct comparison between the attend-to-face vs. ignore-faces condition was used to estimate the occurrence of competitive interactions between simultaneously encoded faces. Neural competition in the ignorefaces condition was investigated in more detail by comparing conditions where similar and dissimilar pairs of faces were presented. The similarity manipulation was also used to test the hypothesis that a higher overlap in representation (for similar faces) corresponded to a higher level of competition.

According to BC, we expected and observed a lower fMRI signal change when the two faces were ignored compared to when one of them was attended to. In addition, and most interestingly, we found an attention $\times$ similarity interaction 


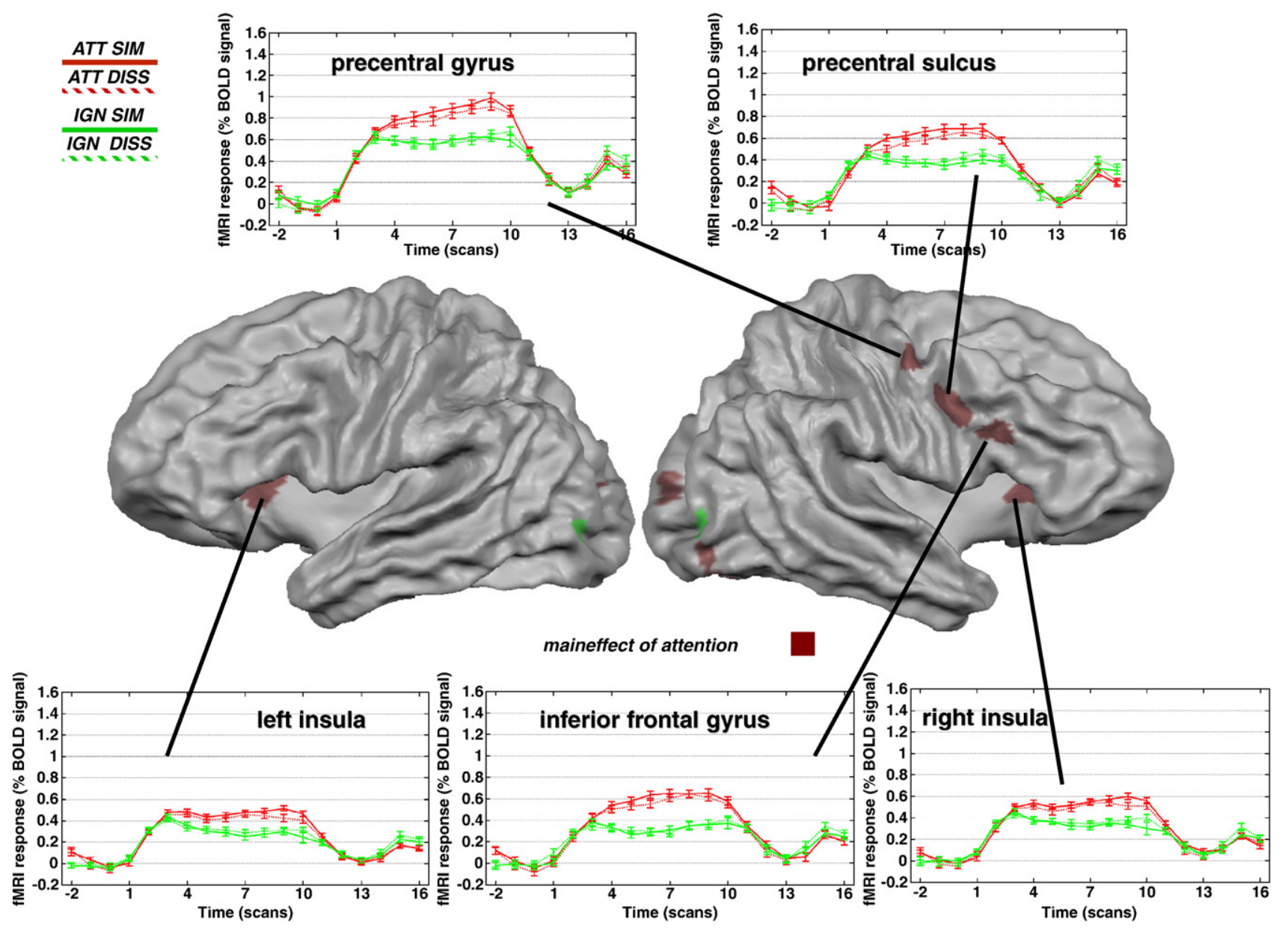

Fig. 2 - Results of GLM analysis in frontal areas (whole brain analysis), separately for left and right hemisphere. The mean time courses of the fMRI signal change per condition and per region of interest are displayed. The error bars in the mean time courses represent the standard error (SE).

effect in FFA and OFA suggesting that the similarity manipulation influenced neural activations only within the ignorefaces condition but not in the attend-to-face condition. The direction of the similarity effect in the ignore-faces condition (similar < dissimilar) can be interpreted within the framework of BC and neural population coding: similar faces compete for representation more than dissimilar faces because they are encoded by neurons that are spatially clustered together.

In line with the bias effect of the BC model we also observed that the enhancement of activation observed during the attend-to-face condition was independent of the similarity level between the faces (similar=dissimilar). We interpreted these results as support for the BC model for face selection within face selective areas. Finally, results in frontal areas suggested that the attentional bias might be controlled by the precentral gyrus/sulcus and the IFG.

\subsection{Neural competition and similarity modulation in FFA and OFA}

In the classical BC paradigm, neural competition in early visual areas between two stimuli was normally tested by comparing the activation of those items presented alone with the same items presented simultaneously. However, the higher amount of transients for single faces presented in sequence compared to pairs of faces presented simultaneously could give rise to an overestimation of neural competition occurring in the latter condition. The attend-to-face condition, in contrast, had the same amount of transients as the ignore-faces condition, and hence offers an alternative way to study BC. In addition, cell recording and fMRI studies found that, when attention was directed to one of the items presented in a multiple display, the neural response increased up to the level of the response to the single item presented alone (Recanzone, 2000; Reynolds et al., 1999). A recent fMRI study confirmed this idea in the face perception domain as it showed that the attend-to-face condition is a suitable baseline for single-face processing (Reddy et al., 2009).

Therefore, in order to overcome the problem of differences in stimulation onsets, we used the comparison attend-to-face vs. ignore-faces condition to investigate neural competition in FFA. We also relied on the assumption that FFA is sensitive to faces presented in two different hemifields (Halgren et al., 1999; Reinholz and Pollmann, 2007) and that, in turn, both faces were encoded by the same cells and compete for representation (Desimone, 1998). As expected, the fMRI signal was lower when both faces in the pair were ignored compared to when one of them was attended (ignore-faces < attend-to-face) suggesting that two faces simultaneously presented mutually interact with each 
other in a suppressive manner. However, this difference between the two tasks could also be related to an enhancement effect due to spatial attention.

In order to confirm the hypothesis that faces in a pair compete for representation, we focused on the influence of the similarity manipulation within the ignore-faces condition. In line with the neural competition effect, we observed that the similarity level between the two faces played a role only within the ignore-faces and not in the attend-to-face condition. In particular, the ROI analysis in FFA revealed a significant interaction of the factors attention (ATT vs. IGN) and similarity (SIM vs. DISS) and a significant modulation by similarity within the ignore-faces condition. In line with our predictions, fMRI signal change in FFA was smaller for similar than for dissimilar faces when both faces were ignored. We interpreted this result as reflecting greater neural competition between similar faces. A vast line of research investigated the spatial organization in primate inferior temporal (IT) cortex and its functional role in object recognition. Several electrophysiological and optical imaging experiments revealed a general tendency for similar complex features to be mapped in nearby locations in IT (Fujita et al., 1992; Kreiman et al., 2006; Sato et al., 2008; Tamura et al., 2005; Tanaka, 2003; Wang et al., 1998). In humans, two fMRI experiments confirmed these findings in the context of face processing (Jiang et al., 2006; Loffler et al., 2005). Both studies used an fMRI adaptation (AMRI-A) paradigm to infer functional properties of neural populations at subvoxel resolutions (GrillSpector et al., 2006). Results from both studies suggested that similar faces were encoded by the same neural population while dissimilar faces were encoded by different subpopulations.

The proposed spatial organization of face selective areas may play a role during neural competition among simultaneously presented stimuli. In fact, the different level of similarity between faces in our experiment would draw upon different spatial distributions of neurons encoding both faces in FFA. In particular, we assumed that similar faces would share their neural representations within the same neural population and they would be seen as ambiguous input at the neural level. This would imply in the context of BC that two similar faces simultaneously presented compete more for representation than dissimilar faces. The higher amount of competitive interactions, in the case of similar faces, should result in a lower fMRI signal change in FFA. The significant pairwise comparison IGN_SIM $<$ IGN_DISS supported this hypothesis. In a more general context, this finding of a visual similarity effect also indicates that both faces were processed by FFA to some extent when presented at the same time even though ignored.

We observed the same results in OFA as we found in FFA. According to the model of face perception of Haxby et al. (2000) face information is processed within two stages. In the first stage, individual facial features and specific attributes as age or gender are extracted (Bruce and Young, 1986; Sergent et al., 1992). Information from the first stage is sent further to the second processing stage, in which global face configuration is achieved. The first processing stage has been associated with OFA, which sends information along the ventral stream to FFA, which has been associated with the second processing stage. Based on this model, we can conclude that neural competition between face representations occurred also at the level of single face features in OFA.
Next to neural coding, perceptual grouping may also play a role in explaining the data in the context of BC. More specifically, an interaction of grouping and $\mathrm{BC}$ might account for the similarity modulation we observed in FFA and OFA in the ignore-faces condition (interaction of attention $\times$ similarity). In fact, we might assume that similar faces form a perceptual group according to the Gestalt laws whereas dissimilar faces do not. In a review on BC, Reynolds and Desimone (1999) proposed a link between neural competition and perceptual grouping. They claimed that items forming perceptual groups are encoded within the same receptive field. Therefore, as competitive interactions are stronger at the level of receptive field, two similar faces (grouping by similarity) were more likely to compete for representation than dissimilar faces, leading to a lower fMRI signal. In the same review, Reynolds and Desimone also suggested that attending to one stimulus cause the RF of a cell to "shrink" around the attended item only. This in turn would explain the absence of similarity modulation within the attend-to-face condition. However, for the current study we consider the grouping interpretation as rather unlikely because of the low amount of stimuli (only two faces), their relatively large size $\left(6.5^{\circ} \times 5.4^{\circ}\right.$ each) and their position in the visual filed (two opposite hemifield).

As an alternative to the BC account, our result could be related to neural adaptation. Several fMRI studies have shown a decreased BOLD response following stimulus repetition. This effect has normally been tested by presenting sequences of similar or identical items. In our study we also mentioned two fMRI studies that performed an adaptation design using similar faces. Both experiment showed a reduced activity when similar faces were presented compared to dissimilar faces, demonstrating that FFA might be functionally organized according to the similarity level of the faces represented. Three models have been proposed as a neural basis for the adaptation effect. The fatigue model assumes that the same neurons activated during the first presentation are also activated but less strongly when the stimulus is presented again. In the sharpening model, neurons become specialized and during the repetition a smaller percentage of neurons activates compared to the first stimulus presentation. Finally, the facilitation model predicts that repetition of a stimulus causes faster processing of the stimulus repeated which leads a neural activity of shorter duration and/or latencies (GrillSpector et al., 2006). To our knowledge, none of these neural causes have been related to the effects normally observed when several items are simultaneously presented. In addition the present experiment was carefully designed in order to avoid any adaptation effect due to repetition of similar faces. In fact, the similarity level between faces was manipulated only at the level of single trial (within a pair) and not across trials. Pairs of faces in a block were different to each other both in the similar and dissimilar condition such that any type of adaptation across conditions was kept constant. However, due to the limited understanding of human neural adaptation, we cannot strictly rule out that similar mechanisms (fatigue, sharpening, or facilitation) are effective in sequential as well as in simultaneous presentation.

A third possible interpretation of the results in the ignorefaces condition could be that neurons processing one of the faces were inhibited via lateral local connections by surrounding neurons encoding the other face. This, in turn, would 
result in a higher inhibition effect for similar compared to dissimilar faces (Knierim and van Essen, 1992).

\subsection{Attentional bias in FFA and OFA}

The involvement of spatial attention to one of the faces elicited a general enhancement of hemodynamic activity compared to the line comparison task (Wojciulik et al., 1998). Furthermore, the enhancement we observed when attention was shifted to one of the faces was statistically indistinguishable when the distractor was similar or dissimilar to the target face. Therefore, attention to the target face seems to increase the effective "spatial resolution" of the visual system in order to process only the face attended among the two presented (Reynolds and Desimone, 1999). Moreover, the same level of activation between the two attend-to-face conditions confirmed that single faces in both similar and dissimilar sets were properly selected to elicit the same fMRI signal change in FFA. It is unlikely that the increased activation in FFA and OFA was due to a higher level of difficulty of the attend-to-face task compared to the ignore-faces task. In fact, the behavioral results showed no significant differences in reaction time and error rates between the two tasks. The amount of eye movement recorded in an EEG experiment using the same experimental design partially confirmed these results. In fact, the total amount of horizontal saccades was very low $(<4 \%)$ and a two-tailed ANOVA did not show any difference between the two tasks (attend-to-face, ignore-faces conditions). However, if we assumed that in the attend-to-face task subjects had the tendency to make horizontal eye-movements towards the face on the left, a one-tailed ANOVA was the proper statistical test to perform. This analysis revealed a significantly larger amount of horizontal saccades for the attend-toface vs. ignore-faces condition. Therefore, we cannot fully rule out the possibility that the attentional effect we found in OFA and FFA was not related to eye-movement.

In summary, in line with the BC model, results in FFA and OFA suggested that the two simultaneously presented faces were encoded and compete with each other in these areas (attend-to-face >ignore-faces, ignore-dissimilar-faces $>$ ignoresimilar-faces). In addition, we observed that attention solved neural competition by biasing the neural processing towards the target face only (attend-to-face>ignore-faces, attend-todissimilar-face $=$ attend - to-similar-face) .

\subsection{Attentional bias via frontal areas}

Results in visual areas showed that when one of the faces in the visual field was attended, fMRI signal change increased compared to the neural response during the ignore-faces condition. The whole brain analysis revealed possible frontal sources of this biasing signal. In particular, the insula, IFG, precentral sulcus and precentral gyrus were selectively recruited during the attentional task. In the present attentional task the subjects attended to the face on the left and performed a match-to-face task. The task was performed while ignoring a distracting face on the right. Previous studies involving similar attentional tasks found an increase of activation in ventrolateral prefrontal regions (Gitelman et al., 1999; Hopfinger et al., 2000; Thomsen et al., 2005). According to the authors these areas were involved in the inhibitory filtering of the distracting item in the hemifield opposite to the focus of attention. In a slightly different view Brown et al. (2006) proposed that both the insula and IFG are needed to keep attention captured while inhibiting any shift of attention towards the distracting location. Our results for the insula and IFG might reflect both mechanisms. In fact, in order to perform the task, subjects optimized their focus on the "target" position (left) by suppressing any attentional bias to the right. Activation in the insula during an attentional task might also reflect a higher level of anxiety or alertness, as proposed by Lawrence et al. (2003) in a rapid visual information processing paradigm.

The right precentral sulcus and gyrus also showed a higher fMRI signal change during the attentional task compared to the ignore-faces task. Although the precentral sulcus has been demonstrated to be recruited during generation of eye movement, several studies showed that this area is also involved in visuospatial attentional processes (Brown et al., 2006; Corbetta et al., 1998; Gitelman et al., 1999; Grosbras et al., 2005; Hopfinger et al., 2000; Law et al., 1997; Nobre et al., 1997; Thomsen et al., 2005) and attentional control (Pessoa et al., 2003). This versatile nature of the precentral sulcus was already hypothesized by Rizzolatti et al. (1987) within the premotor theory of attention. He stated that covert attentional mechanisms and overt saccade generation share a neural circuitry in the initial stages of processing.

Together with the precentral sulcus, the precentral gyrus is also part of the network involved in saccadic eye movement and covert attention (Grosbras et al., 2005; Hopfinger et al., 2000; Law et al., 1997; Petit et al., 1999). In summary, the right precentral sulcus/gyrus and IFG seem to be plausible candidates to be involved in the bias of neural competition in face selective areas. Overall, this study showed for the first time that BC may play a relevant role in FFA and OFA for face selection.

\section{Experimental procedures}

\subsection{Subjects}

Nineteen healthy and paid volunteers (10 females, one lefthanded) with normal or corrected to normal visual acuity participated in this study (mean age $=25.3$ years, $S D=1.8$ years). After explanation of the procedures, participants signed an informed consent form. The study was approved by the ethical committee of the faculty. In 16 subjects (out of 19) FFA was properly localized. As FFA was the main focus of the present investigation, the remaining participants (3 out 19) were discarded from further analysis. Due to technical difficulties of the button-box, the behavioral responses were recorded for 15 subjects (in the final set of 16).

\subsection{Stimulus preparation}

The stimuli consisted of two sets of 16 pairs of faces. One set represented similar (SIM) and the other dissimilar (DISS) faces (Figs. 3c, 4b). The faces in the pairs were selected from a large set of pictures of faces (total of 60 pictures) provided by Mark Steyvers (http://psiexp.ss.uci.edu/research/software.htm). The pictures were all taken under similar lightning with neutral facial expressions (Kayser, 1985). 
a)

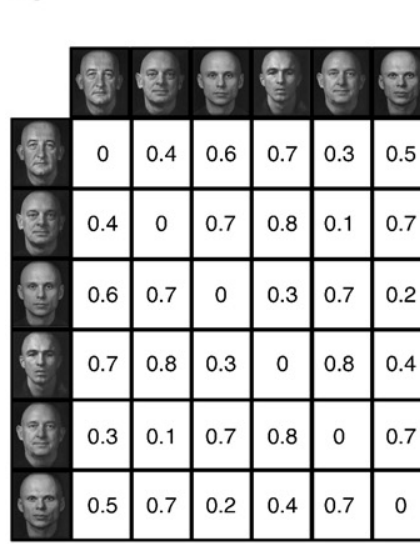

b)

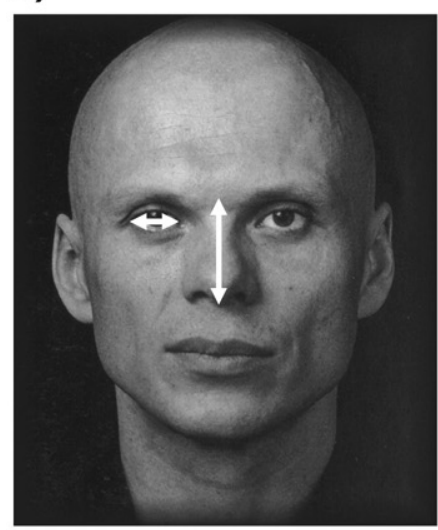

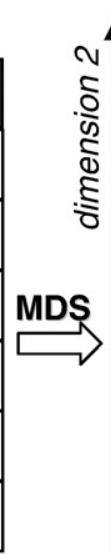


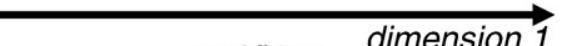

reference

face

c)


Fig. 3 - An example of material selection of a similar and dissimilar pair of faces given one face (reference face). (a) Top-down approach of selection: a multidimensional space of faces was build up from a dissimilarity matrix (the higher the number the lower the similarity between two faces) via multidimensional scaling technique (MDS). The Euclidean distance between the reference face and the others (mutual distance) was computed as a measure of similarity degrees. (b) Bottom-up approach of selection: 30 physical measures for each face (only 2 of them displayed in the figure) were represented in a multidimensional space. The similarity degree between the two faces was measured as in the top-down approach. In the bottom-up approach two dissimilar faces had to be at the same distance from the mean face. (c) Result of selection: combination of results from both approaches.

Similarity between faces was defined by using the two major approaches adopted for face representation: the purely psychological or top-down approach and the purely computational or bottom-up approach (Fig. 3a, b). Both approaches are based on the multidimensional representation of faces (Steyvers and Busey, 2000). In the psychological approach, the data for computing two-face similarity consisted of similarity ratings of each face with all the other faces. Similarities or dissimilarities in these data were explored using scaling techniques. A Multidimensional Scaling algorithm (MDS) assigned to each face a location in a multidimensional space (Steyvers, 2002) (Fig. 3a). We first computed the Euclidean distance between a given face (i.e. its Cartesian representation in the multidimensional space) and all the others resulting in a "mutual distance" value (Fig. 3a, b). Secondly, two faces were considered SIM (or DISS), for this specific approach, if their mutual distance was smaller (or larger) than a specific threshold set according to the overall distribution of the mutual distances. In particular, two faces were labeled as similar if the mutual distance between two faces was smaller than the mean of the mutual distance distribution plus 0.70 times its standard deviation. Similarly, if the mutual distance was larger than the mean of the mutual distance distribution plus one time its standard deviation two faces were labeled as dissimilar.

In the computational approach, a face was represented by a collection of features or dimensions (e.g. nose length, width of the eye brows, etc.) explicitly derived from a 2D image of the face (Fig. 3b). The same two-step procedure as described above, for the psychological approach, was used to define pairs of SIM and DISS faces. However, for the latter set an additional constraint was considered. We took into account the observation that faces are encoded by their direction (facial identity) and distance (distinctiveness) from a 


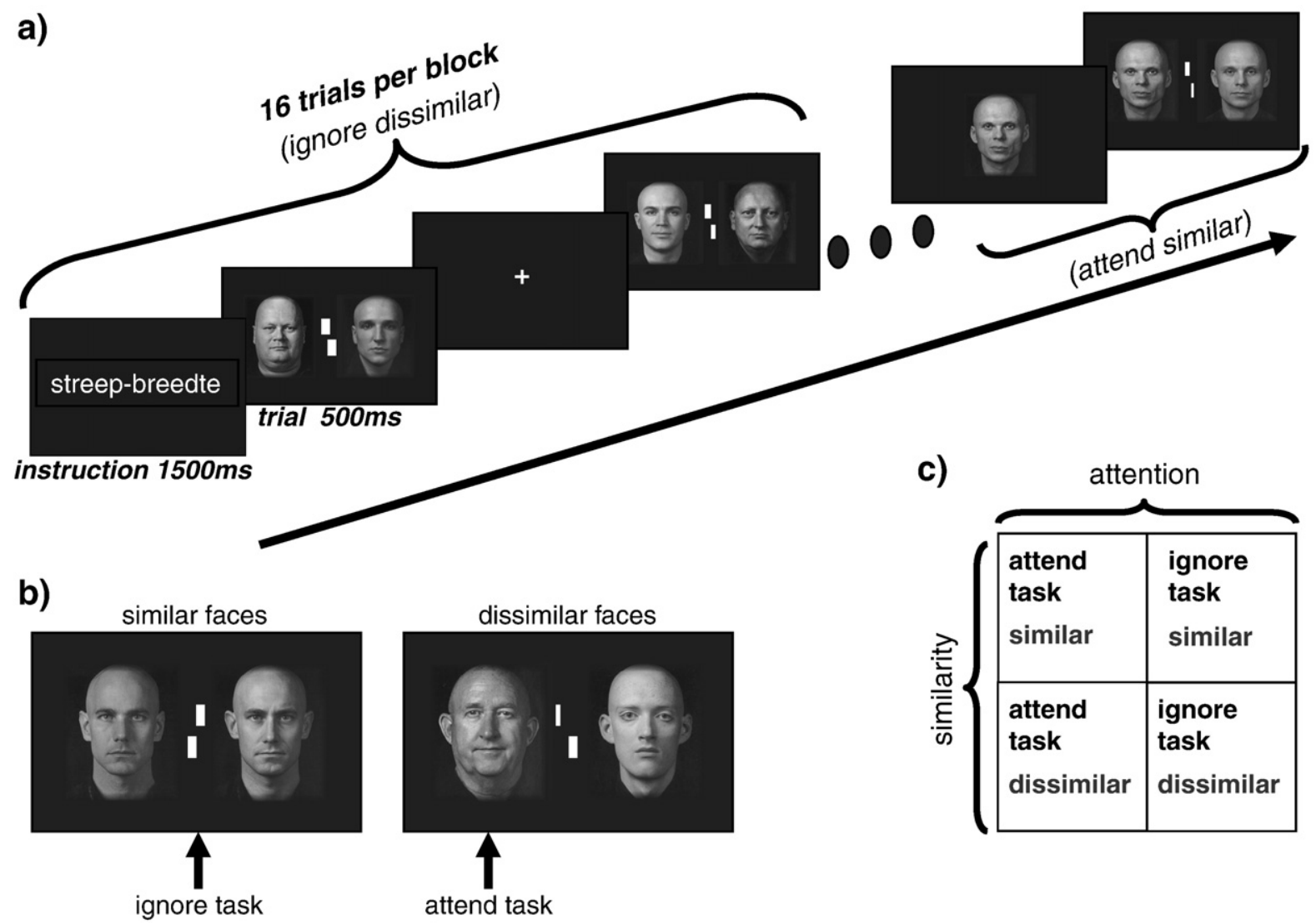

Fig. 4 - Experimental design and settings. (a) Block structure with examples of two different conditions (ignore-faces with dissimilar display and attend-to-face with similar display). (b) Examples of the two types of the display presented (similar and dissimilar faces) for the two tasks (attend-to-face and ignore-faces). (c) Conditions: $2 \times 2$ repeated measures design.

prototypical (mean) face (Loffler et al., 2005). According to this study, visual stimulation of a particular face elicits a different level of activation in FFA depending on its position in a multidimensional space from the mean face. Therefore, in order to control that both faces in the pair equally activate FFA, two faces were considered DISS only if (a) their mutual distance was larger than a specific threshold (see above), and if (b) they both differed in the same amount from the mean face. The mean face was computed from all the faces used in the experiment.

The final set of stimuli was derived as follows: a pair of faces was labeled as a SIM (or DISS) pair, only if both faces were labeled as such in the top-down as well as in the bottom-up approach (Fig. 3c). As a last step, we computed the distance of both sets of faces from the mean face in order to avoid differences in the level of activation within FFA, across conditions. SIM and DISS faces were, on average, at the same distance from the mean face.

\subsection{Procedure in the main experiment}

The main experiment consisted of two runs in a block-design (Fig. 4a). Each run consisted of 32 blocks and started with a blank screen with a white cross in the centre lasting for 18 seconds. A block consisted of sixteen trials. A single trial started with a $500 \mathrm{~ms}$ of blank screen, followed by a pair of faces presented for $500 \mathrm{~ms}$. The two faces were positioned in opposite hemifields and they were either SIM or DISS to each other (see Stimulus preparation). The single pictures subtended approximately $6.5^{\circ}$ (height) $\times 5.4^{\circ}$ (width) of visual angle and were aligned with the centre of the screen. The entire visual display covered $6.5^{\circ}$ (height) $\times 12.2^{\circ}$ (width) of visual angle. The display size was designed to be comparable with those used in previous studies on face processing (Gauthier et al., 2000; Halgren et al., 1999; Kanwisher et al., 1997; Rossion et al., 2003; Steeves et al., 2006; Wojciulik et al., 1998). It is also reasonable to assume that FFA integrates the two simultaneously presented faces within each experimental trial (Halgren et al., 1999; Reinholz and Pollmann, 2007). Between the faces, two bars $\left(0.2^{\circ}\right.$ in height) were presented $0.15^{\circ}$ above and below the centre of the visual screen. A block of trials ended with a resting condition consisting of a blank screen lasting for $10 \mathrm{~s}$.

A visual instruction was presented at the beginning of each block for $1500 \mathrm{~ms}$, and signalled the type of task to be performed in the following block. For the ignore-faces (IGN) task, two keywords ("streep-breedte," "line-width" in English) centred on the screen, instructed the subject to perform a bar-width discrimination task in the centre of the display, while ignoring the background (Fig. 4b). More specifically, participants had to attend to the two bars in the centre and a button-press was required when the bars matched in width (2-3 targets per block). 
The width of the bars varied between 1 and 5 pixels. For the attend-to-face (ATT) task, the instruction consisted of a picture of a face, on which the subject had to perform a match-tosample task. For each trial, participants were asked to respond via a button press when the face presented on the left side of the screen matched the one presented in the instruction phase (Fig. 4b). For this task, subjects were instructed beforehand to avoid saccades and to covertly attend to the item on the left while fixating the centre. Within each block, targets (matches) occurred on average $15 \%$ of the time (randomly varied between $12.50 \%$ and $18.75 \%$ across blocks and participants). In analogy to preceding biased competition studies (Bles et al., 2006) targets always occurred at the target position, on the left side of the visual field. In both tasks, the time for the subject to respond was $1000 \mathrm{~ms}$ (trial duration).

The design was a $2 \times 2$ repeated measures design with factors task (ATT, IGN) and similarity (SIM, DISS) leading to 4 conditions (IGN_DISS, IGN_SIM, ATT_SIM, ATT_DISS, Fig. 4c). The participants were not made aware of the similarity manipulation. The order of the blocks was randomised and counterbalanced across participants and runs by means of Williams design (Williams, 1949). This scheme is a generalized Latin square design, balanced for first-order carryover effects. The stimulus display was identical for both types of task. Participants were instructed to fixate the centre of the screen throughout the experiment and to minimise head and eyemovements while in the scanner.

\subsection{Data acquisition}

Images were acquired on a $3 \mathrm{~T}$ Siemens Magnetom Allegra head scanner (Siemens Medical System, Erlangen, Germany) using a standard head coil. Thirty-two oblique axial slices (inplane resolution: $3.5 \mathrm{~mm} \times 3.5 \mathrm{~mm}$, slice thickness: $3.5 \mathrm{~mm}$, interslice distance $0 \mathrm{~mm}$ ) covering the entire cortical volume were acquired using an echo planar imaging sequence (repetition time $(\mathrm{TR})=2000 \mathrm{~ms}$, echo time $(\mathrm{TE})=29 \mathrm{~ms}$, matrix: $64 \times 64)$. We acquired 612 volumes per run and discarded the first two from the analysis due to the T1 saturation effect. Functional slices were aligned to a high resolution 3D anatomical dataset acquired in the middle of the entire session and consisting of 176 slices (for 15 subjects the MDEFT sequence was used: $T R=7.92 \mathrm{~ms}$; $\mathrm{TE}=2.4 \mathrm{~ms}$; flip angle $=15^{\circ}$, voxel dimension $=1 \times 1 \times 1 \mathrm{~mm}^{3}$; due to better performances the ADNI sequence replaced the MDEFT sequence for 4 subjects: $\mathrm{TR}=2250 \mathrm{~ms}$; $\mathrm{TE}=2.6 \mathrm{~ms}$; flip angle $=9^{\circ}$, voxel dimension $=1 \times 1 \times 1 \mathrm{~mm}^{3}$ ).

The participants were placed comfortably in the scanner and their head was fixated with foam pads. Participants saw the stimuli projected on a screen through a mirror mounted on the head coil. The visual field was perceived at a distance of 57 centimeters. The stimuli were delivered using the Presentation 12.2 package (Neurobehavioral Systems, Inc. www.neurobs.com). Stimulus presentation was synchronized with MR data acquisition by triggering the stimulus program with the first MR pulse.

\subsection{Analysis}

Both the functional and the anatomical data were analyzed using the BrainVoyager QX package (Brain Innovation B.V.,
Maastricht, The Netherlands). The anatomical scans were used to project the statistical results from the functional data onto high-resolution anatomical images. They were also used to derive the Talairach coordinates needed to normalize both sets of data.

Functional data were pre-processed, aligned to the anatomical images, and normalized. The pre-processing procedure started with correcting the data for motion artefacts in three dimensions and for slice scan-time differences. Subsequently, linear drifts were removed from the signal and data were high-pass filtered to remove slow frequency drifts up to 9 cycles per time course. After the pre-processing, functional data were aligned to the high-resolution anatomical images and normalized to the standard 3-dimensional Talairach space. The final version of the functional data consisted of a 4-dimensional $(x, y, z, t)$ dataset in Talairach space for each run and participant.

After brain normalization, the statistical analysis from multiple subjects was performed via the GLM. In the main experiment, an RFX approach was used with subject as a random variable. The RFX-GLM analysis was implemented via multilevel summary statistics. In a first level, a standard GLM analysis was used to estimate the beta values (summary statistics) for each subject. These values were entered as dependent variables in the second level analysis. At the second analysis level full factorial effects (main effects and $2 \times 2$ interaction effect) were tested. These effects were tested in conjunction with contrasting all the conditions versus baseline. The statistical analysis was carried out in a ROI, which was specified by an independent functional localizer (FFA, see below), and in the whole brain.

For the whole-brain analysis, correction for multiple comparisons was performed using cluster-size thresholding. The procedure started with thresholding the map relative to the contrast of interest with $t=3.15(p=.007)$ as suggested by Goebel et al. (2006) at the voxel-level. Next, 1000 normally distributed noise images were generated by randomly selecting values from a normal distribution (Monte Carlo simulation), and the noise map was smoothed using an estimate of the intrinsic smoothness of the source statistical map (Fororman et al., 1995; Goebel et al., 2006). The noise images were thresholded at the pre-defined voxel-level threshold. Clusters of voxels surviving the threshold were tabulated according to cluster size. The final distribution of cluster sizes was thresholded against a false-positive rate of 0.05 . The minimum cluster size that survived the thresholding was used as cluster-size threshold of the source statistical map. This yielded a multiple comparison correction of $p=0.05$ at the cluster-level. The correction for multiple comparisons was not performed for the ROI analysis in FFA. In fact, in this case, the beta values of the fMRI signal change per subject and condition were extracted, averaged over all voxels in the single-subject volume-of-interest (VOI), and evaluated (summary statistics, level 1).

Statistical maps were projected on an average brain, which was computed by averaging normalized cortices from all the subjects. The normalization of the cortices was achieved via cortex-based alignment. This procedure aligns the individual cortical renderings by increasing the overlap of curvature information of the cortices (Fischl et al., 1999; Goebel et al., 2006). 


\subsection{Localization of FFA}

An independent functional run, consisting of 324 volumes, was used to localize FFA in all subjects. The scannerparameters used to acquire the data were the same used for the main experimental runs. The stimuli used to localize the face-processing-area consisted of pictures of faces, scrambled faces, objects, and places (Goffaux et al., 2009). The scrambled faces were obtained by applying the Fourier phase randomization procedure (Nasanen, 1999) to the original set of faces. The algorithm for "scrambling" faces consisted of two steps: each picture from the set of faces was first Fourier-transformed. Subsequently, the phase spectrum of the resulted transform was replaced by random values, while keeping the amplitude spectrum of the image unaltered. This procedure yielded images that preserved the global low-level properties of the original image (luminance, contrast, spectral energy, etc.), and degraded completely any category-related information.

The 4 different categories of stimuli were presented in blocks (20 stimuli per block) for 4 times. In order to keep the attention of the subject constant across the run, participants were asked to indicate with a button-press whether the stimulus colour changed from a typical black-white to a light red.

After the acquisition, the functional data were pre-processed (in this case, slow frequency drifts were removed up to 3 cycles) and normalized following the same procedure previously described for the main experimental runs. For the statistical analysis of the FFA localizer, a FFX-GLM analysis was performed with the four stimulus types as predictors. The condition faces was contrasted with all the others. Finally, FFA was localized considering the clusters of voxels, within the fusiform gyrus, statistically significant (FDR-corrected, $q=.05$ ). For 2 subjects (out of 16 analyzed) the left hemisphere did not reveal any FFA localization. Therefore, for the main experiment, the ROI analysis was performed for 16 subjects in the right FFA and for 14 subjects in the left FFA (Table 1).

\section{Acknowledgments}

The authors wish to thank M. Bles for helpful discussions. This project was supported by NWO ASPASIA grant nr. 015.001.053 and the Maastricht University HRM Policy "Mobilizing minds" (increase of females in higher positions).

\section{REFERENCES}

Beck, D.M., Kastner, S., 2005. Stimulus context modulates competition in human extrastriate cortex. Nat. Neurosci. 8, 1110-1116.

Beck, D.M., Kastner, S., 2007. Stimulus similarity modulates competitive interactions in human visual cortex. J. Vis. 7 (19), 11-12.

Bentin, S., Allison, T., Puce, A., Perez, E., McCarthy, G., 1996. Electrophysiological studies of face perception in humans. J. Cogn. Neurosci. 551-565.

Bles, M., Schwarzbach, J., De Weerd, P., Goebel, R., Jansma, B.M., 2006. Receptive field size-dependent attention effects in simultaneously presented stimulus displays. Neuroimage 30, 506-511.

Brefczynski, J.A., DeYoe, E.A., 1999. A physiological correlate of the "spotlight" of visual attention. Nat. Neurosci. 2, 370-374.

Broadbent, D., 1958. Perception and communication Pergamon. Elmsford, New York.

Brown, M.R., Goltz, H.C., Vilis, T., Ford, K.A., Everling, S., 2006. Inhibition and generation of saccades: rapid event-related fMRI of prosaccades, antisaccades, and nogo trials. Neuroimage 33, 644-659.

Bruce, V., Young, A., 1986. Understanding face recognition. Br. J. Psychol. 77 (Pt 3), 305-327.

Carrasco, M., Ling, S., Read, S., 2004. Attention alters appearance. Nat. Neurosci. 7, 308-313.

Chelazzi, L., Duncan, J., Miller, E.K., Desimone, R., 1998. Responses of neurons in inferior temporal cortex during memory-guided visual search. J. Neurophysiol. 80, 2918-2940.

Chelazzi, L., Miller, E.K., Duncan, J., Desimone, R., 2001. Responses of neurons in macaque area V4 during memory-guided visual search. Cereb. Cortex 11, 761-772.

Corbetta, M., Akbudak, E., Conturo, T.E., Snyder, A.Z., Ollinger, J.M., Drury, H.A., Linenweber, M.R., Petersen, S.E., Raichle, M.E., Van Essen, D.C., Shulman, G.L., 1998. A common network of functional areas for attention and eye movements. Neuron 21, 761-773.

Corbetta, M., Miezin, F.M., Shulman, G.L., Petersen, S.E., 1993. A PET study of visuospatial attention. J. Neurosci. 13, 1202-1226.

Deffke, I., Sander, T., Heidenreich, J., Sommer, W., Curio, G., Trahms, L., Lueschow, A., 2007. MEG/EEG sources of the 170-ms response to faces are co-localized in the fusiform gyrus. Neuroimage 35, 1495-1501.

Desimone, R., 1998. Visual attention mediated by biased competition in extrastriate visual cortex. Philos. Trans. R. Soc. Lond. B Biol. Sci. 353, 1245-1255.

Desimone, R., Duncan, J., 1995. Neural mechanisms of selective visual attention. Annu. Rev. Neurosci. 18, 193-222.

Dubois, S., Rossion, B., Schiltz, C., Bodart, J.M., Michel, C., Bruyer, R., Crommelinck, M., 1999. Effect of familiarity on the processing of human faces. Neuroimage 9, 278-289.

Fischl, B., Sereno, M.I., Tootell, R.B., Dale, A.M., 1999. High-resolution intersubject averaging and a coordinate system for the cortical surface. Hum. Brain Mapp. 8, 272-284.

Forman, S.D., Cohen, J.D., Fitzgerald, M., Eddy, W.F., Mintun, M.A., Noll, D.C., 1995. Improved assessment of significant activation in functional magnetic resonance imaging (fMRI): use of a cluster-size threshold. Magn. Reson. Med. 33, 636-647.

Fujita, I., Tanaka, K., Ito, M., Cheng, K., 1992. Columns for visual features of objects in monkey inferotemporal cortex. Nature 360, 343-346.

Gauthier, I., Tarr, M.J., Moylan, J., Skudlarski, P., Gore, J.C., Anderson, A.W., 2000. The fusiform "face area" is part of a network that processes faces at the individual level. J. Cogn. Neurosci. 12, 495-504.

Gitelman, D.R., Nobre, A.C., Parrish, T.B., LaBar, K.S., Kim, Y.H., Meyer, J.R., Mesulam, M., 1999. A large-scale distributed network for covert spatial attention: further anatomical delineation based on stringent behavioural and cognitive controls. Brain 122 (Pt 6), 1093-1106.

Goebel, R., Esposito, F., Formisano, E., 2006. Analysis of functional image analysis contest (FIAC) data with brainvoyager QX: from single-subject to cortically aligned group general linear model analysis and self-organizing group independent component analysis. Hum. Brain Mapp. 27, 392-401.

Goffaux, V., Rossion, B., Sorger, B., Schiltz, C., Goebel, R., 2009. Face inversion disrupts the perception of vertical relations between features in the right human occipito-temporal cortex. J. Neuropsychol. 3, 45-67. 
Grill-Spector, K., Henson, R., Martin, A., 2006. Repetition and the brain: neural models of stimulus-specific effects. Trends Cogn. Sci. 10, 14-23.

Grill-Spector, K., Knouf, N., Kanwisher, N., 2004. The fusiform face area subserves face perception, not generic within-category identification. Nat. Neurosci. 7, 555-562.

Grosbras, M.H., Laird, A.R., Paus, T., 2005. Cortical regions involved in eye movements, shifts of attention, and gaze perception. Hum. Brain Mapp. 25, 140-154.

Halgren, E., Dale, A.M., Sereno, M.I., Tootell, R.B., Marinkovic, K., Rosen, B.R., 1999. Location of human face-selective cortex with respect to retinotopic areas. Hum. Brain Mapp. 7, 29-37.

Hawkins, H.L., Hillyard, S.A., Luck, S.J., Mouloua, M., Downing, C.J., Woodward, D.P., 1990. Visual attention modulates signal detectability. J. Exp. Psychol. Hum. Percept. Perform. 16, 802-811.

Haxby, J.V., Gobbini, M.I., Furey, M.L., Ishai, A., Schouten, J.L., Pietrini, P., 2001. Distributed and overlapping representations of faces and objects in ventral temporal cortex. Science 293, 2425-2430.

Haxby, J.V., Hoffman, E.A., Gobbini, M.I., 2000. The distributed human neural system for face perception. Trends Cogn. Sci. 4, 223-233.

Haxby, J.V., Ungerleider, L.G., Clark, V.P., Schouten, J.L., Hoffman, E.A., Martin, A., 1999. The effect of face inversion on activity in human neural systems for face and object perception. Neuron 22, 189-199.

Hillyard, S.A., Munte, T.F., 1984. Selective attention to color and location: an analysis with event-related brain potentials. Percept. Psychophys. 36, 185-198.

Hoffman, E.A., Haxby, J.V., 2000. Distinct representations of eye gaze and identity in the distributed human neural system for face perception. Nat. Neurosci. 3, 80-84.

Hopfinger, J.B., Buonocore, M.H., Mangun, G.R., 2000. The neural mechanisms of top-down attentional control. Nat. Neurosci. 3, 284-291.

Jacques, C., Rossion, B., 2004. Concurrent processing reveals competition between visual representations of faces. NeuroReport 15, 2417-2421.

Jacques, C., Rossion, B., 2006. The time course of visual competition to the presentation of centrally fixated faces. J. Vis. 6, 154-162.

Jiang, X., Rosen, E., Zeffiro, T., Vanmeter, J., Blanz, V., Riesenhuber, M., 2006. Evaluation of a shape-based model of human face discrimination using FMRI and behavioral techniques. Neuron 50, 159-172.

Kanwisher, N., McDermott, J., Chun, M.M., 1997. The fusiform face area: a module in human extrastriate cortex specialized for face perception. J. Neurosci. 17, 4302-4311.

Kastner, S., De Weerd, P., Desimone, R., Ungerleider, L.G., 1998. Mechanisms of directed attention in the human extrastriate cortex as revealed by functional MRI. Science 282, 108-111.

Kastner, S., De Weerd, P., Pinsk, M.A., Elizondo, M.I., Desimone, R., Ungerleider, L.G., 2001. Modulation of sensory suppression: implications for receptive field sizes in the human visual cortex. J. Neurophysiol. 86, 1398-1411.

Kastner, S., Pinsk, M.A., De Weerd, P., Desimone, R., Ungerleider, L.G., 1999. Increased activity in human visual cortex during directed attention in the absence of visual stimulation. Neuron 22, 751-761.

Kastner, S., Ungerleider, L.G., 2000. Mechanisms of visual attention in the human cortex. Annu. Rev. Neurosci. 23, 315-341.

Kastner, S., Ungerleider, L.G., 2001. The neural basis of biased competition in human visual cortex. Neuropsychologia 39, 1263-1276.

Kayser, S., 1985. Heads. Abbeville Press, New York.

Knierim, J.J., van Essen, D.C., 1992. Neuronal responses to static texture patterns in area V1 of the alert macaque monkey. J. Neurophysiol. 67, 961-980.
Kreiman, G., Hung, C.P., Kraskov, A., Quiroga, R.Q., Poggio, T., DiCarlo, J.J., 2006. Object selectivity of local field potentials and spikes in the macaque inferior temporal cortex. Neuron 49, 433-445.

Law, I., Svarer, C., Holm, S., Paulson, O.B., 1997. The activation pattern in normal humans during suppression, imagination and performance of saccadic eye movements. Acta Physiol. Scand. 161, 419-434.

Lawrence, N.S., Ross, T.J., Hoffmann, R., Garavan, H., Stein, E.A., 2003. Multiple neuronal networks mediate sustained attention. J. Cogn. Neurosci. 15, 1028-1038.

Loffler, G., Yourganov, G., Wilkinson, F., Wilson, H.R., 2005. fMRI evidence for the neural representation of faces. Nat. Neurosci. 8, 1386-1390.

Luck, S.J., Chelazzi, L., Hillyard, S.A., Desimone, R., 1997. Neural mechanisms of spatial selective attention in areas V1, V2, and V4 of macaque visual cortex. J. Neurophysiol. 77, 24-42.

Luck, S.J., Hillyard, S.A., Mouloua, M., Woldorff, M.G., Clark, V.P., Hawkins, H.L., 1994. Effects of spatial cuing on luminance detectability: psychophysical and electrophysiological evidence for early selection. J. Exp. Psychol. Hum. Percept. Perform. 20, 887-904.

Miller, E.K., Gochin, P.M., Gross, C.G., 1993. Suppression of visual responses of neurons in inferior temporal cortex of the awake macaque by addition of a second stimulus. Brain Res. 616, $25-29$.

Moran, J., Desimone, R., 1985. Selective attention gates visual processing in the extrastriate cortex. Science 229, 782-784.

Nasanen, R., 1999. Spatial frequency bandwidth used in the recognition of facial images. Vis. Res. 39, 3824-3833.

Nobre, A.C., Sebestyen, G.N., Gitelman, D.R., Mesulam, M.M., Frackowiak, R.S., Frith, C.D., 1997. Functional localization of the system for visuospatial attention using positron emission tomography. Brain 120 (Pt 3), 515-533.

Op de Beeck, H.P., Dicarlo, J.J., Goense, J.B., Grill-Spector, K., Papanastassiou, A., Tanifuji, M., Tsao, D.Y., 2008. Fine-scale spatial organization of face and object selectivity in the temporal lobe: do functional magnetic resonance imaging, optical imaging, and electrophysiology agree? J. Neurosci. 28, 11796-11801.

Pessoa, L., Kastner, S., Ungerleider, L.G., 2003. Neuroimaging studies of attention: from modulation of sensory processing to top-down control. J. Neurosci. 23, 3990-3998.

Petit, L., Dubois, S., Tzourio, N., Dejardin, S., Crivello, F., Michel, C., Etard, O., Denise, P., Roucoux, A., Mazoyer, B., 1999. PET study of the human foveal fixation system. Hum. Brain Mapp. 8, 28-43.

Posner, M.I., Snyder, C.R., Davidson, B.J., 1980. Attention and the detection of signals. J. Exp. Psychol. 109, 160-174.

Recanzone, G.H., 2000. Spatial processing in the auditory cortex of the macaque monkey. Proc. Natl. Acad. Sci. U. S. A. 97, 11829-11835.

Reddy, L., Kanwisher, N.G., VanRullen, R., 2009. Attention and biased competition in multi-voxel object representations. Proc. Natl. Acad. Sci. U. S. A. 106, 21447-21452.

Reinholz, J., Pollmann, S., 2007. Neural basis of redundancy effects in visual object categorization. Neurosci. Lett. 412, 123-128.

Reynolds, J.H., Chelazzi, L., Desimone, R., 1999. Competitive mechanisms subserve attention in macaque areas V2 and V4. J. Neurosci. 19, 1736-1753.

Reynolds, J.H., Desimone, R., 1999. The role of neural mechanisms of attention in solving the binding problem. Neuron 24 (19-29), 111-125.

Reynolds, J.H., Pasternak, T., Desimone, R., 2000. Attention increases sensitivity of V4 neurons. Neuron 26, 703-714.

Rizzolatti, G., Riggio, L., Dascola, I., Umilta, C., 1987. Reorienting attention across the horizontal and vertical meridians: evidence in favor of a premotor theory of attention. Neuropsychologia 25, $31-40$. 
Rossion, B., Caldara, R., Seghier, M., Schuller, A.M., Lazeyras, F., Mayer, E., 2003. A network of occipito-temporal face-sensitive areas besides the right middle fusiform gyrus is necessary for normal face processing. Brain 126, 2381-2395.

Rossion, B., Delvenne, J.F., Debatisse, D., Goffaux, V., Bruyer, R., Crommelinck, M., Guerit, J.M., 1999. Spatio-temporal localization of the face inversion effect: an event-related potentials study. Biol. Psychol. 50, 173-189.

Sato, T., Uchida, G., Tanifuji, M., 2008. Cortical columnar organization is reconsidered in inferior temporal cortex. Cereb. Cortex.

Sergent, J., Ohta, S., MacDonald, B., 1992. Functional neuroanatomy of face and object processing. A positron emission tomography study. Brain 115 (Pt 1), 15-36.

Steeves, J.K., Culham, J.C., Duchaine, B.C., Pratesi, C.C., Valyear, K.F., Schindler, I., Humphrey, G.K., Milner, A.D., Goodale, M.A., 2006. The fusiform face area is not sufficient for face recognition: evidence from a patient with dense prosopagnosia and no occipital face area. Neuropsychologia 44, 594-609.

Steyvers, M., 2002. Multidimensional scaling. Encyclopedia of Cognitive Science. Nature Publishing Group, London, UK.

Steyvers, M., Busey, T., 2000. Predicting similarity ratings to faces using physical descriptions. Computational, geometric, and process perspectives on facial cognition: contexts and challenges. Lawrence Erlbaum Associates.
Tamura, H., Kaneko, H., Fujita, I., 2005. Quantitative analysis of functional clustering of neurons in the macaque inferior temporal cortex. Neurosci. Res. 52, 311-322.

Tanaka, K., 2003. Columns for complex visual object features in the inferotemporal cortex: clustering of cells with similar but slightly different stimulus selectivities. Cereb. Cortex 13, 90-99.

Thomsen, T., Specht, K., Ersland, L., Hugdahl, K., 2005. Processing of conflicting cues in an attention-shift paradigm studied with fMRI. Neurosci. Lett. 380, 138-142.

Treisman, A.M., 1969. Strategies and models of selective attention. Psychol. Rev. 76, 282-299.

Treue, S., Martinez Trujillo, J.C., 1999. Feature-based attention influences motion processing gain in macaque visual cortex. Nature 399, 575-579.

Treue, S., Maunsell, J.H., 1996. Attentional modulation of visual motion processing in cortical areas MT and MST. Nature 382, 539-541.

Wang, G., Tanifuji, M., Tanaka, K., 1998. Functional architecture in monkey inferotemporal cortex revealed by in vivo optical imaging. Neurosci. Res. 32, 33-46.

Williams, E.J., 1949. Experimental designs balanced for the estimation of residual effects of treatments. Aust. J. Sci. Res. 2, 149-168.

Wojciulik, E., Kanwisher, N., Driver, J., 1998. Covert visual attention modulates face-specific activity in the human fusiform gyrus: fMRI study. J. Neurophysiol. 79, 1574-1578. 